Check for updates

Cite this: RSC Adv., 2017, 7, 32592

\title{
In situ structural modification of graphitic carbon nitride by alkali halides and influence on photocatalytic activity
}

\author{
Yan Xu, Yinyan Gong, DD* Hui Ren, Wenbo Liu, Lengyuan Niu, Can Li and Xinjuan Liu
}

A series of graphitic carbon nitride photocatalysts were prepared by thermally polymerizing the mixtures of melamine and alkali halides ( $\mathrm{MCl}, \mathrm{M}=\mathrm{Na}, \mathrm{K}, \mathrm{Rb}, \mathrm{Cs}$ ) in air. XRD, FTIR, Raman, SEM and TEM were used to investigate the effect of alkali halides on the structure and morphology of $\mathrm{g}-\mathrm{C}_{3} \mathrm{~N}_{4}$. It is found that such prepared samples exhibit reduced in-plane long-range periodic ordering while maintaining the framework of melon strands. However, no significant increase in surface area was observed by BET measurements. Moreover, PL and EIS measurements show that alkali halide modification results in improved separation and transfer of photogenerated electron and holes. Furthermore, the results of UV-vis absorption spectra and ultraviolet photoelectron spectra (UPS) indicate that the presence of alkali halides during the thermal polymerization process can decrease band gap energy and shift the conduction/valence bands of $g-C_{3} N_{4}$ upward. The variation of structural, electronic, and optical properties depends on the type of cationic ions. Potassium chloride modification gives rise to the best photocatalytic performance among all the samples, which is attributed to the reduced charge carrier recombination, narrower band gap, and higher CB edge. These findings may help in the design of visible-light-driven photocatalysts with enhanced photocatalytic activity.

Received 17th May 2017

Accepted 19th June 2017

DOI: $10.1039 / \mathrm{c} 7 \mathrm{ra05555b}$

rsc.li/rsc-advances
Graphitic carbon nitride, a polymeric semiconductor material, appears as an intriguing visible-light-driven photocatalyst due to the moderate band gap $\left(E_{\mathrm{g}}=2.7 \mathrm{eV}\right)$, and suitable band edge positions. ${ }^{1} \mathrm{~g}-\mathrm{C}_{3} \mathrm{~N}_{4}$ is commonly prepared by thermal condensation of organic precursors containing both carbon and nitrogen and pristine g- $\mathrm{C}_{3} \mathrm{~N}_{4}$ typically has a multilayered structure with weak interlayer van der Waals interaction and strong intralayer covalent bonding. ${ }^{10}$ However, graphitic carbon nitride usually encounters problems such as high recombination rate of photogenerated charge carriers and a low amount of surface active sites, and thus has limited photocatalytic efficiency. To address this issue, different strategies have been proposed including doping, ${ }^{11-13}$ manipulating microstructure and morphology, ${ }^{14-19}$ coupling with other semiconductors and noble metals ${ }^{8,20-24}$ and isotype g- $\mathrm{C}_{3} \mathrm{~N}_{4} / \mathrm{g}$ $\mathrm{C}_{3} \mathrm{~N}_{4}$ junctions. ${ }^{17}$

Considering its unique structure, post-growth thermal treatment is frequently used to modify the structural and photocatalytic properties of $\mathrm{g}-\mathrm{C}_{3} \mathrm{~N}_{4} \cdot{ }^{15,16,25}$ For instance, porous structures $^{26}$ and nanosheets ${ }^{15,16,25}$ with different surface functional groups have been prepared by thermal exfoliating bulk $\mathrm{g}$ $\mathrm{C}_{3} \mathrm{~N}_{4}$ in different atmosphere (e.g. air, $\mathrm{NH}_{3}$, and oxygen gas). In addition to interrupting the periodic stacking of carbon nitride monolayer in the vertical direction, researchers also try to break the in-plane long-range arrangement of melon units. It has been proposed $^{27-29}$ that incomplete deamination of amine-groupcontaining precursors can result in the formation of abundant hydrogen bonds in the covalent bonding dominated intralayer framework, i.e. the basal plane can be considered as strands of
Center for Coordination Bond Metrology and Engineering, College of Materials Science and Engineering, China Jiliang University, Hangzhou, Zhejiang 310018, China. E-mail: ygong2007@gmail.com 
polymeric melon units connected by hydrogen bonds. Kang et al. ${ }^{27}$ demonstrated that post-growth annealing of bulk g- $\mathrm{C}_{3} \mathrm{~N}_{4}$ in inert ambient can selectively break these hydrogen bonds and, consequently, enhance the photocatalytic activity due to faster transfer of charge carriers across plane, suppressed recombination of charge carriers and increased visible light absorption.

Alkali metal halides have been used previous to enhance the photocatalytic activity of $\mathrm{g}-\mathrm{C}_{3} \mathrm{~N}_{4} \cdot{ }^{30-34}$ For example, $\mathrm{Hu}$ et al. prepared K-doped $\mathrm{g}-\mathrm{C}_{3} \mathrm{~N}_{4}$ by using dicyanadiamide and potassium hydrate as precursors, and such obtained photocatalysts have tunable band edge positions and enhanced mineralization ability. ${ }^{31}$ Zhang et al. studied K-doped g- $\mathrm{C}_{3} \mathrm{~N}_{4}$ synthesized by thermal polymerization of dicyanadiamide and $\mathrm{KI}$, and found that the photocatalytic efficiency for phenol and $\mathrm{MB}$ degradation was about 3.3 and 5.8 times that of bulk g- $\mathrm{C}_{3} \mathrm{~N}_{4}$, respectively. ${ }^{32}$ Moreover, isotype triazine-heptazine based $\mathrm{g}-\mathrm{C}_{3} \mathrm{~N}_{4}$ heterojunctions were synthesized in the media of $\mathrm{KCl} / \mathrm{LiCl}$ salts, and the photocatalytic hydrogen generation rate is 23 times that of pristine $\mathrm{g}-\mathrm{C}_{3} \mathrm{~N}_{4} \cdot{ }^{34}$ As neither complicated post-growth treatment process nor hazardous chemicals are involve, this ecofriendly method of employing alkali metal halides to improve the photocatalytic performance of $\mathrm{g}-\mathrm{C}_{3} \mathrm{~N}_{4}$ appears intriguing. However, little attention has been given to investigate the influence of different alkali ions on the structural, electronic, optical, and photocatalytic properties of $\mathrm{g}-\mathrm{C}_{3} \mathrm{~N}_{4}$, which will be helpful to select appropriate alkali halides and develop photocatalysts with enhanced photocatalytic performance.

Herein, we synthesized a series of samples using melamine and alkali halides ( $\mathrm{MCl}, \mathrm{M}=\mathrm{Na}, \mathrm{K}, \mathrm{Rb}$, and $\mathrm{Cs}$ ) as precursors. XRD, FTIR, Raman, SEM and TEM measurements were carried out to investigate the effect of various cationic ions on the structural and morphological properties of $\mathrm{g}-\mathrm{C}_{3} \mathrm{~N}_{4}$. Moreover, the chemical state of alkali ions and the salt-induced variations of electronic and optical properties of $\mathrm{g}-\mathrm{C}_{3} \mathrm{~N}_{4}$ photocatalysts were studied in detail by X-ray photoemission (XPS), UV-visible diffuse reflectance (DRS), ultraviolet photoelectron (UPS), photoluminescence (PL), and electrochemical impedance (EIS) spectra. Finally, the photocatalytic performance was evaluated by degradation of RhB under visible light irradiation, and the effects of different cationic ions were discussed.

\section{Experimental}

\section{Materials}

Melamine (CP, Sinopharm Chemical Reagent Co. Ltd), $\mathrm{NaCl}$ (AR, Sinopharm Chemical Reagent Co. Ltd), KCl (AR, Aladdin Industrial Corporation), $\mathrm{RbCl}$ (AR, Aladdin Industrial Corporation), CsCl (AR, Aladdin Industrial Corporation), and Rhodamine $\mathrm{B}$ (RhB, AR, Aladdin Industrial Corporation). All the chemicals were used as received without further purification, and deionized water was used during all the experiments.

\section{Synthesis}

A series of $\mathrm{g}-\mathrm{C}_{3} \mathrm{~N}_{4}$ samples were prepared using melamine and alkali halides as precursors. ${ }^{35}$ Typically, $1.5 \mathrm{~g}$ of melamine and
$3.75 \mathrm{~g}$ of alkali halides ( $\mathrm{MCl}, \mathrm{M}=\mathrm{Na}, \mathrm{K}, \mathrm{Rb}, \mathrm{Cs}$ ) were fully mixed by grinding in an agate mortar for $15 \mathrm{~min}$. The mixture was then transferred into a ceramic crucible covered by a lid and calcinated in a muffle furnace at $550{ }^{\circ} \mathrm{C}$ for $4 \mathrm{~h}$ at a ramping rate of $2{ }^{\circ} \mathrm{C} \min ^{-1}$. After being naturally cooled down to room temperature, such obtained products were ground into powder, dispersed in $100 \mathrm{~mL}$ DI water and stirred for $4 \mathrm{~h}$. And then, the suspension was centrifuged, washed by water for 3 times, and dried in an oven at $80{ }^{\circ} \mathrm{C}$ for $12 \mathrm{~h}$. The collected photocatalysts were labeled as GCN-Na, GCN-K, GCN-Rb and GCN-Cs corresponding to $\mathrm{NaCl}, \mathrm{KCl}, \mathrm{RbCl}$ and $\mathrm{CsCl}$, respectively. For comparison, pristine $\mathrm{g}-\mathrm{C}_{3} \mathrm{~N}_{4}$ was synthesized by heating $1.5 \mathrm{~g}$ melamine at $550{ }^{\circ} \mathrm{C}$ for $4 \mathrm{~h}(\mathrm{GCN}-0)$.

\section{Characterization}

The structure and morphology of all the samples were characterized by XRD (Rigaku D/Max-2400 diffractometer, Japan), FTIR (Thermal Fisher Nicolet i50, US), Raman spectra (inVia, Renishaw, UK) under $785 \mathrm{~nm}$ excitation, SEM (SU8010 FE-SEM, Hitachi, Japan) and TEM (JEM-2100 F, JEOL, Japan). The BrunauerEmmett-Teller (BET) surface area was determined by nitrogen adsorption-desorption isotherms measured on a Micromeritics ASAP2460 system at $77 \mathrm{~K}$. PL spectra were also recorded on inVia spectrometer using the luminescence module under the excitation of $325 \mathrm{~nm}$ UV laser. XPS measurements were performed on ESCALAB $250 \mathrm{Xi}$ (Thermal Scientific, UK) using Al-K $\alpha$ radiation and binding energies were referenced to $\mathrm{C} 1 \mathrm{~s}$ line at $284.8 \mathrm{eV}$ from adventitious carbon. The DRS spectra were measured on a UV-VisNIR spectrometer equipped with integrated sphere (Lamada 750S, Perkin Elmer, US). The UPS measurements were carried out on a home-built system with HeII $(40.8 \mathrm{eV})$ emission line employed for excitation. A sample bias of $-5 \mathrm{~V}$ was applied to observe the secondary electron cutoff (SEC). EIS measurements were carried out on an electrochemical working station (CHI660E, Shanghai, China) in $1 \times 10^{-5} \mathrm{M} \mathrm{RhB}$ aqueous solution using a threeelectrode configuration under daylight. A Pt foil and a standard calomel electrode were served as the counter electrode and the reference electrode, respectively. The working electrodes were prepared by mixing $25 \mathrm{mg}$ photocatalysts in $0.2 \mathrm{~mL} 2.5 \%$ polyvinyl alcohol binder, and the obtained slurry was then coated on a $1 \mathrm{~cm}$ $\times 2.5 \mathrm{~cm}$ ITO slice with an effective working area of $1 \mathrm{~cm}^{2}$ by a screen printing method. After that, the electrodes were dried in a vacuum oven at $80{ }^{\circ} \mathrm{C}$ for $12 \mathrm{~h}$. For the EIS measurements, the applied bias voltage and ac amplitude were set at open-circuit voltage and $10 \mathrm{mV}$ with the frequency varying in the range of 0.1 Hz-1 MHz.

\section{Photocatalytic degradation of $\mathrm{RhB}$}

$50 \mathrm{mg}$ photocatalysts were dispersed in $50 \mathrm{~mL}$ of $\mathrm{RhB}\left(2 \times 10^{-5}\right.$ $\mathrm{M})$ in a quartz tube and magnetically stirred for $60 \mathrm{~min}$ in dark to reach an adsorption-desorption equilibrium. A halogen lamp, power regulated at $500 \mathrm{~W}$ and equipped with a $400 \mathrm{~nm}$ cut-off filter, was used as light source. The lamp was placed vertically in a double-wall jacketed quartz tube with cooling water circulated to maintain a nearly constant temperature during photocatalytic reaction. $4 \mathrm{~mL}$ of suspension was 
withdrawn every $10 \mathrm{~min}$, and the absorbance of RhB solution was measured by Lamada $750 \mathrm{~S}$ spectrometer at $554 \mathrm{~nm}$ after centrifugation. A recycled photocatalytic activity test was carried out according to the above-mentioned procedure. After each run of photocatalytic reaction, the photocatalyst was collected, washed thoroughly by deionized water and dried. To investigate the photocatalytic mechanism, trapping experiments were performed to investigate the main active species during the photocatalytic process. Certain amounts of benzoquinone (BQ), isopropanol (IPA), and ammonium oxalate (AO) were introduced in the above suspension of photocatalysts as the scavengers to detect superoxide radicals $\left({ }^{\circ} \mathrm{O}_{2}{ }^{-}\right)$, hydroxyl radicals $\left({ }^{\circ} \mathrm{OH}\right)$, and holes $\left(\mathrm{h}^{+}\right)$, respectively. The photocatalytic degradation of RhB was treated the same as the photodegradation process.

\section{Results and discussion}

As presented in Fig. 1a, the XRD pattern of pristine $\mathrm{g}-\mathrm{C}_{3} \mathrm{~N}_{4}(\mathrm{GCN}-0)$ exhibited two characteristic diffraction peaks at $2 \theta \approx 13.2^{\circ}$ and $2 \theta$ $\approx 27.6^{\circ}$. The former can be assigned to the (100) crystal plane, representing the in-plane long-range periodic packing and the latter is associated with the (002) crystal plane, representing the stacking of carbon nitride monolayers along the $c$-axis..$^{10,14,27,36}$ The absence of $13.2^{\circ}$ peak for GCN-Na, GCN-K, GCN-Rb, and GCN-Cs suggests that $\mathrm{g}-\mathrm{C}_{3} \mathrm{~N}_{4}$ synthesized in the media of alkali halides has reduced in-plane ordering. Similar results have been obtained for $\mathrm{g}-\mathrm{C}_{3} \mathrm{~N}_{4}$ prepared by incorporating salts and bases such as $\mathrm{KI},{ }^{32}$
$\mathrm{KCl}^{33}$ and $\mathrm{KOH}^{36}$ or subjected to post-growth thermal annealing in inert ambient. ${ }^{27}$ It has been proposed that the intralayer framework of carbon nitride is composed of covalent-bond-dominated polymeric melon strands interconnected by hydrogen bonds. ${ }^{10,27}$ As the strength of hydrogen bonds is relatively weaker than that of covalent bonds between carbon and nitrogen atoms, it is feasible to selectively break the hydrogen bonds and disturbs in-plane stacking $^{27,28}$ For our samples, it is plausible that the presence of alkali halides during thermal polymerization of melamine suppresses the formation of hydrogen bonding between melon units, and thus reduces the intra-layer periodic ordering. Regarding to the $27.6^{\circ}$ peak, the usage of alkali halides shifts the diffraction angle toward the higher angle side and the extent of variation depends on the type of cationic ions. $2 \theta$ of GCN-Na is only slightly increased compared to GCN-0 while a more pronounced shifting of $2 \theta$ from $27.8^{\circ}$ to $28.4^{\circ}$ and $28.2^{\circ}$ are observed for GCN-K and GCN-Rb, respectively, suggesting that alkali halides result in decreased inter-layer separation. This is different from the results of alkali ion doping, ${ }^{32,37}$ and chloride doping, ${ }^{38}$ in which interstitial $\mathrm{Na}$ (or $\mathrm{K}$ ) and intercalation $\mathrm{Cl}$ cause enlarged inter-layer distance. This implies that in our samples neither alkali ions nor chloride ions are doped into the carbon nitride lattice. In addition, the overall diffraction intensity of GCN-Na, GCN-K, GCN-Rb and GCNCs are lower than that of pristine $\mathrm{g}-\mathrm{C}_{3} \mathrm{~N}_{4}$, especially for GCN-Cs which has the lowest diffraction intensity. The weakening of overall diffraction intensity implies that the addition of alkali halides deteriorates the crystallinity of $\mathrm{g}-\mathrm{C}_{3} \mathrm{~N}_{4} \cdot{ }^{33,36,37}$ Therefore, the incorporation of $\mathrm{MCl}(\mathrm{M}=\mathrm{Na}, \mathrm{K}, \mathrm{Rb}, \mathrm{Cs})$ during the thermal
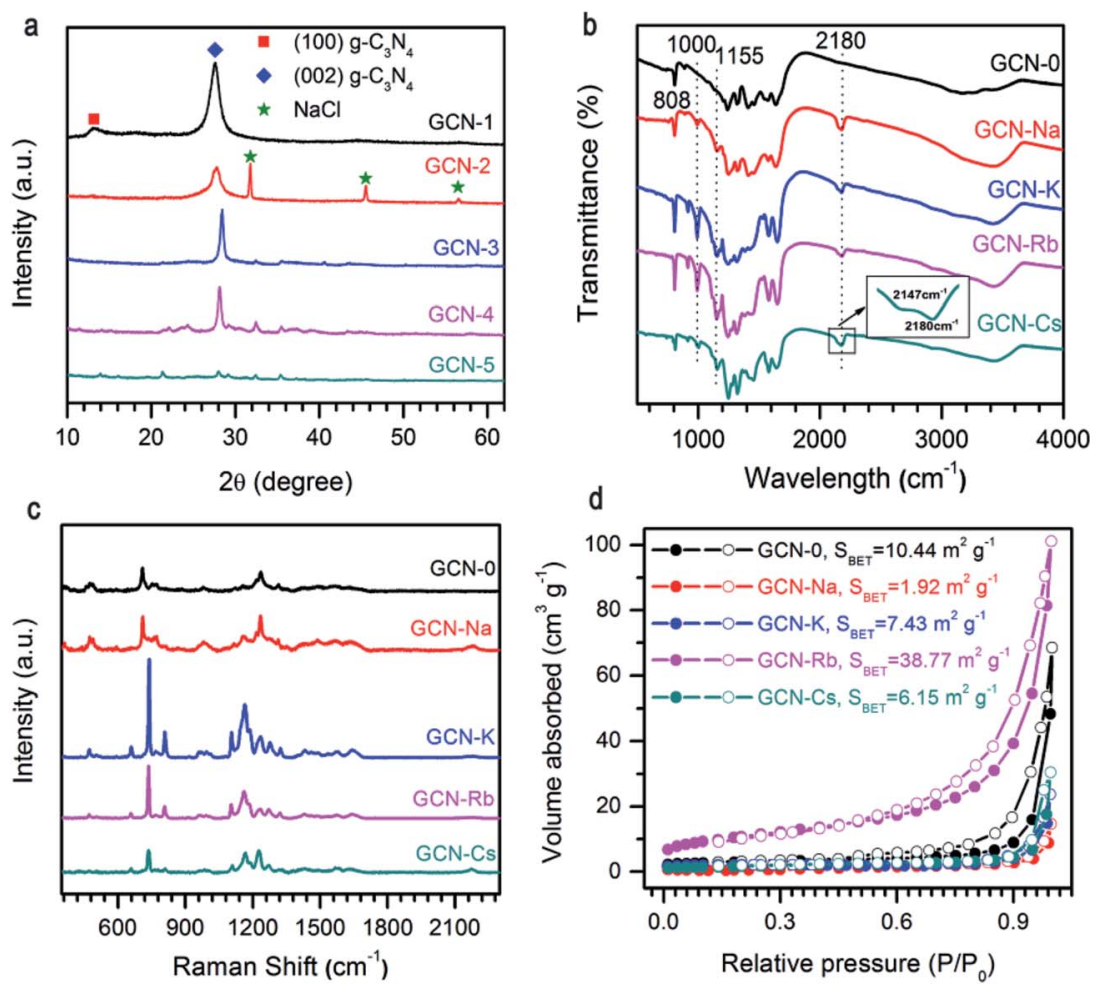

Fig. 1 (a) XRD patterns, (b) FTIR spectra, (c) Raman spectra, and (d) $\mathrm{N}_{2}$ adsorption-desorption isotherms of g- $\mathrm{C}_{3} \mathrm{~N}_{4}$ via $\mathrm{MCl}$ (M = $\mathrm{Na}$, $\mathrm{K}, \mathrm{Rb}, \mathrm{Cs}$ ) modification. Results of pristine $\mathrm{g}-\mathrm{C}_{3} \mathrm{~N}_{4}$ are plotted together for comparison. The extra diffraction peaks for $\mathrm{GCN}-\mathrm{Na}$ in (a) is from $\mathrm{NaCl}$ residual. 
polymerization of melamine can reduce the in-plane long-range ordering, decrease inter-layer spacing and lower the crystallinity of $\mathrm{g}-\mathrm{C}_{3} \mathrm{~N}_{4}$, and the extent of structural modulation is related to the type of cationic ions.

The influence of alkali halides on the structural properties was further investigated by FTIR and Raman spectra due to their high sensitivity to local structure. As shown in Fig. 1b, FTIR spectra of all the samples show comparable characteristic bands at $808 \mathrm{~cm}^{-1}$ and $1200-1900 \mathrm{~cm}^{-1}$ region, which are ascribed to breathing mode of triazine units and stretching vibration modes of aromatic $\mathrm{CN}$ heterocycles, respectively, ${ }^{39,40}$ this confirms that alkali halides only interrupt the intralayer long-range periodic arrangement without modifying the basic atomic building blocks. Compared to pristine $\mathrm{g}_{-} \mathrm{C}_{3} \mathrm{~N}_{4}$, extra peaks at $1000 \mathrm{~cm}^{-1}, 1155 \mathrm{~cm}^{-1}, 2147 \mathrm{~cm}^{-1}$ and 2180 $\mathrm{cm}^{-1}$ are observed for GCN-Na, GCN-K, GCN-Rb and GCN-Cs, which can be attributed to hydroxyl groups grafted on the surface of $\mathrm{g}-\mathrm{C}_{3} \mathrm{~N}_{4}$ (ref. 41 ) and cyano groups $(\mathrm{C} \equiv \mathrm{N}) \cdot{ }^{33,36}$ It is plausible that the presence of alkali ions $\left(\mathrm{Na}^{+}, \mathrm{K}^{+}, \mathrm{Rb}^{+}\right.$, and $\left.\mathrm{Cs}^{+}\right)$could prevent the formation of hydrogen bonds, and then the $-\mathrm{OH}$ groups, dissociated from the $\mathrm{H}_{2} \mathrm{O}$ at high temperatures, will bond to the amino groups presented in $\mathrm{g}-\mathrm{C}_{3} \mathrm{~N}_{4}$. However, a thorough understanding of the underlying mechanism requires a systematic study. ${ }^{10,33,41}$ The broad bands between $3000 \mathrm{~cm}^{-1}$ and $3600 \mathrm{~cm}^{-1}$ are originated from the terminal amino groups. ${ }^{17}$ Vibrations of alkali-related groups are not observed, suggesting that the framework of carbon nitride is not changed after salt treatment. Fig. 1c compares NIR Raman spectra of all the samples. It is found that all the samples have similar Raman spectra, suggesting good retention of microstructure. Two Raman bands with complicated structures at $670-800 \mathrm{~cm}^{-1}$ and $1090-1390 \mathrm{~cm}^{-1}$ might be related to breathing modes of s-triazine. ${ }^{42}$ However, a detailed interpretation of the Raman spectra requires the development of Raman scattering theory of $\mathrm{g}-\mathrm{C}_{3} \mathrm{~N}_{4}$.

Fig. 1d illustrates the $\mathrm{N}_{2}$ adsorption-desorption isotherms of $\mathrm{g}-\mathrm{C}_{3} \mathrm{~N}_{4}$ synthesized from the mixture of melamine and different alkali halides. All the isotherms are type IV, and the specific surface areas calculated by the BET method $\left(S_{\mathrm{BET}}\right)$ are 10.44, $1.92,7.43,38.77$, and $6.15 \mathrm{~m}^{2} \mathrm{~g}^{-1}$ for GCN-0, GCN-Na, GCN-K, GCN-Rb and GCN-Cs, respectively. Except GCN-Rb, all the samples have smaller $S_{\mathrm{BET}}$ than pristine $\mathrm{g}-\mathrm{C}_{3} \mathrm{~N}_{4}$. Results of XRD, FTIR, Raman and BET measurements indicate that the presence of alkali halides serve as template during thermal condensation of melamine and disturb the long-range periodic arrangement of melon strands through hydrogen bonding while maintains the basic atomic units of $\mathrm{C}$ and $\mathrm{N}$ atoms in the basal plane. Cationic ions with larger radius cause more affect the structural variation.

The morphologies of the representative samples were investigated scanning electron microscope (SEM) and transmission electron microscope (TEM) analysis, as shown in Fig. 2. As shown in Fig. 2, the pristine $\mathrm{g}-\mathrm{C}_{3} \mathrm{~N}_{4}$ sample is composed of thick sheets while GCN-K has an irregular porous structure. This phenomenon might be ascribed to the reduced in-plane long-range periodic ordering due to the presence of $\mathrm{KCl}$ during thermal condensation of melamine. TEM images of both samples are shown in Fig. 3, and both samples display the typical lamellar stacking structure.

The specific bonding and surface chemical states of representative samples were investigated by XPS spectra. Fig. 4a illustrates the survey scans of GCN-0 and GCN-K, and the observed $\mathrm{C}, \mathrm{N}$ and $\mathrm{O}$ signals are originate from $\mathrm{g}-\mathrm{C}_{3} \mathrm{~N}_{4}$ and adsorbed $\mathrm{O}$-containing species, respectively. In addition, $\mathrm{K}$ and $\mathrm{Cl}$ are detected in GCN-K, which indicates the existence of $\mathrm{KCl}$ residue. The high-resolution XPS spectra of $\mathrm{N}$ 1s are shown in Fig. $4 \mathrm{~b}$, which can be deconvoluted into three peaks centered at 399.0, 400.4 and $401.6 \mathrm{eV}$, corresponding to the $\mathrm{C}-\mathrm{N}-\mathrm{C}, \mathrm{N}-(\mathrm{C})_{3}$, and $\mathrm{C}-\mathrm{N}-\mathrm{H}$ groups of melon, respectively. ${ }^{34,43,44}$ The $401.6 \mathrm{eV}$ peak shifts to $401.3 \mathrm{eV}$ for $\mathrm{GCN}-\mathrm{K}$, and the decreasing of binding energy might be related to charge redistribution in the framework of $\mathrm{g}-\mathrm{C}_{3} \mathrm{~N}_{4}$ due to microstructure variation by adding $\mathrm{KCl}$.
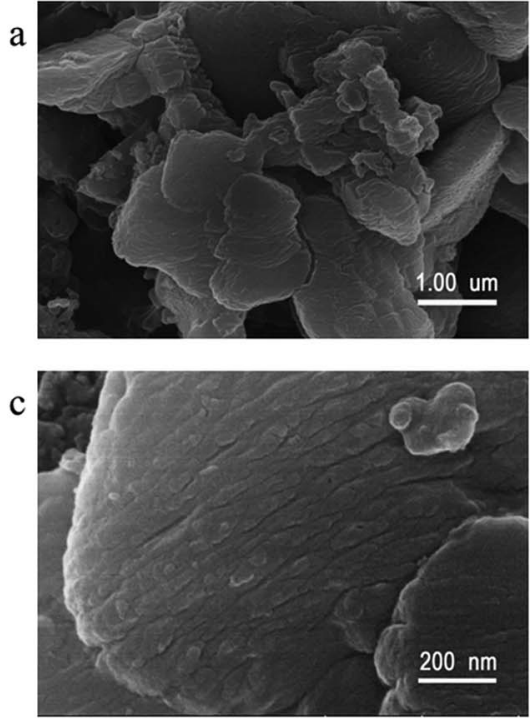

Fig. 2 SEM images of GCN-O (a) and (c), and GCN-K (b) and (d).
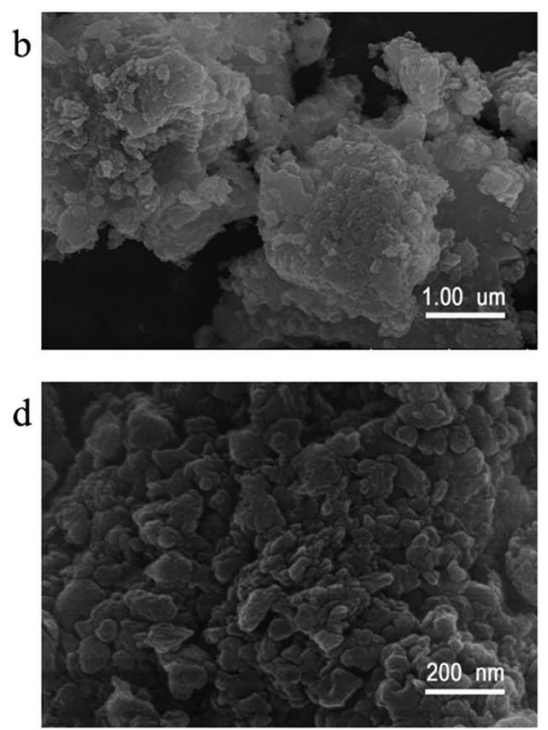

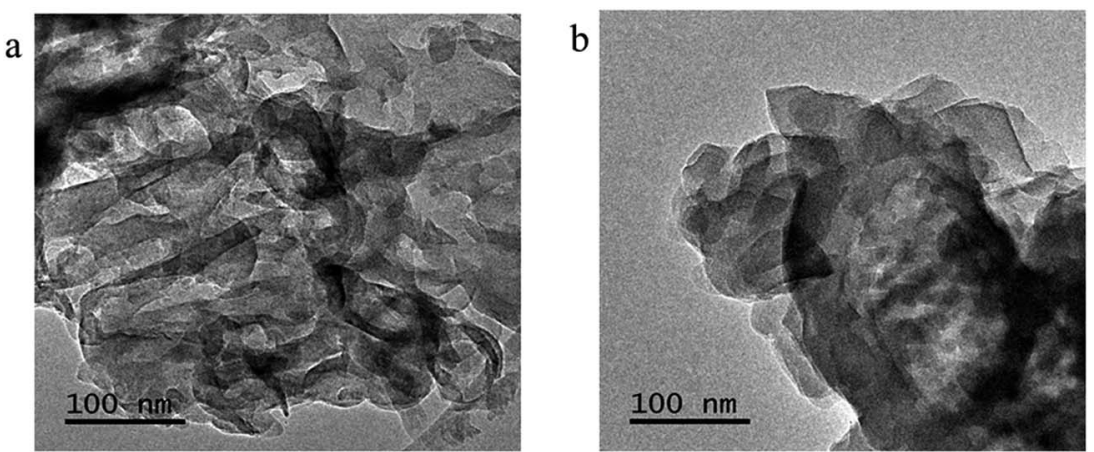

Fig. 3 TEM images of GCN-O (a) and GCN-K (b).
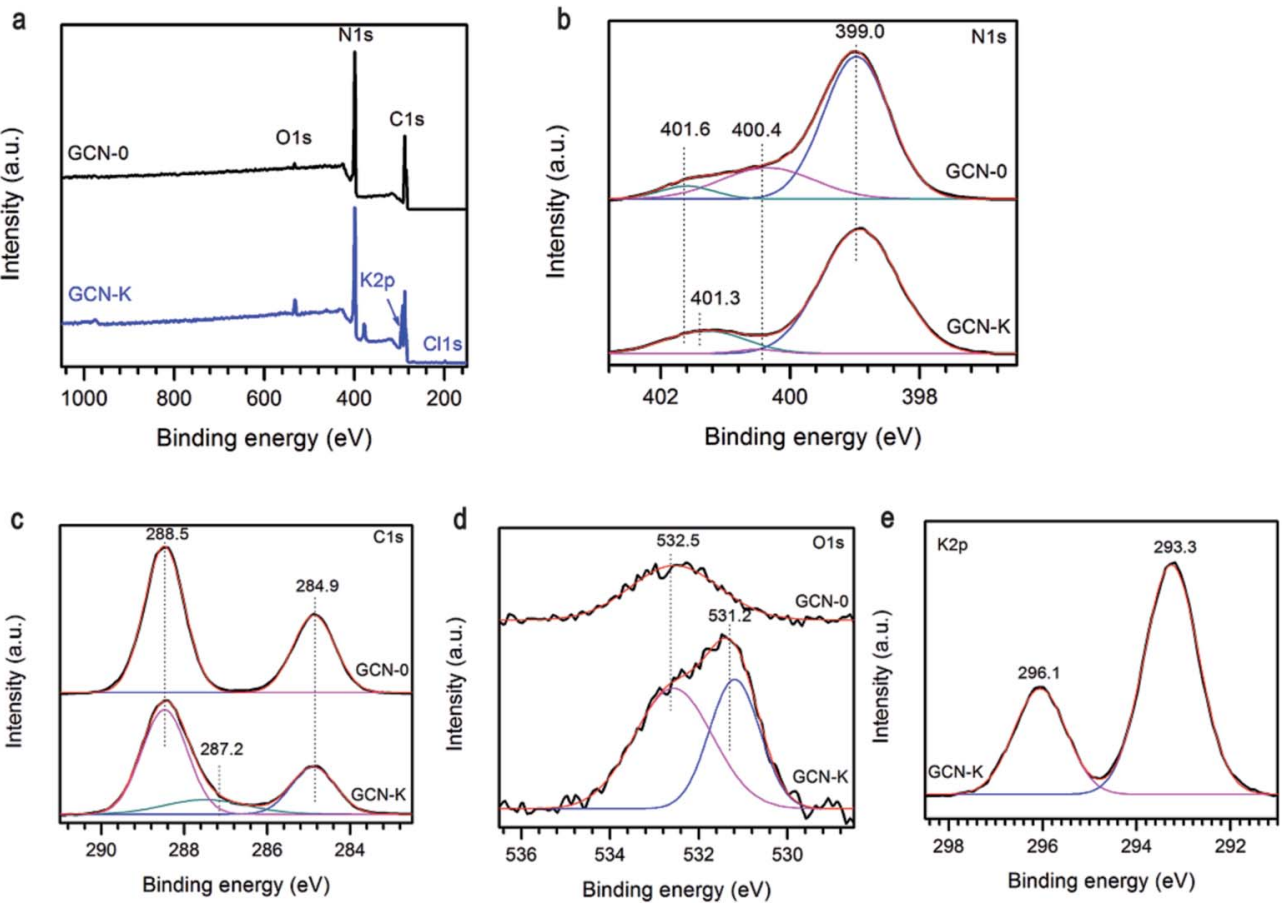

Fig. 4 (a) XPS survey spectra, (b) N 1s, (c) $C 1 s$, (d) $\mathrm{O} 1 \mathrm{~s}$, and (e) $\mathrm{K} 2 \mathrm{p}$ high resolution spectra of $\mathrm{g}-\mathrm{C}_{3} \mathrm{~N}_{4}$ prepared from melamine (GCN-0) and mixture of $\mathrm{KCl}$ and melamine $(\mathrm{GCN}-\mathrm{K})$.

Fig. 4c plots the high-resolution XPS spectra of C 1s, of which there are two peaks at $284.9 \mathrm{eV}$ and $288.5 \mathrm{eV}$, corresponding to $\mathrm{C}==\mathrm{C}$ in adventitious carbon and $\mathrm{C}-\mathrm{N}-\mathrm{C}$ coordination of melon, respectively. ${ }^{27,33,44} \mathrm{An}$ extra peak at $287.2 \mathrm{eV}$ is observed for GCN-K, which is tentatively attributed to $\mathrm{C}-\mathrm{OH}$. Consistent with FTIR result, the presence of alkali halides during thermal condensation facilitate grafting of hydroxyl groups on the surface of $\mathrm{g}-\mathrm{C}_{3} \mathrm{~N}_{4} \cdot{ }^{33,34}$ As shown in Fig. $4 \mathrm{~d}$, the O 1s XPS spectrum of GCN-0 has a single peak at $\sim 532.5 \mathrm{eV}$, which is due to the adsorbed water molecules on the surface of $\mathrm{g}-\mathrm{C}_{3} \mathrm{~N}_{4} \cdot{ }^{33,34,44-46}$ The spectrum of GCN-K can be decomposed into two peaks at $532.5 \mathrm{eV}$ and $531.2 \mathrm{eV}$, corresponding to adsorbed water and N$\mathrm{C}-\mathrm{O}$, respectively. ${ }^{34,46}$ There are two peaks in the $\mathrm{K} 2 \mathrm{p}$ region of GCN-K located at 293.3 and $296.1 \mathrm{eV}$, which is consistent with binding energy of $\mathrm{KCl}^{47}$ The $\mathrm{K} 2 \mathrm{p}$ peaks are higher than the reported values when potassium ions are doped into the interstitial site and form covalent bond with $\mathrm{C}_{3} \mathrm{~N}_{4} \cdot{ }^{32}$ This is consistent with the XRD result.

Fig. 5a plots the UV-visible absorption spectra of all the graphitic carbon nitride samples converted from the measured DRS. Compared to GCN-0, the absorption edges of GCN-Na, GCN-K, GCN-Rb and GCN-Cs were red shifted and the variation follows the sequence $\mathrm{Rb}^{+}>\mathrm{K}^{+}>\mathrm{Na}^{+}<$except for $\mathrm{Cs}^{+}$, i.e. larger cationic ions have more pronounced effect. Steeper absorption edges of GCN-K, GCN-Rb and GCN-Cs than GCN0 and GCN-Na might be due to the higher degree of structure modification. Band gap energies of semiconductors can be estimated using the Kubelka-Munk function., ${ }^{3,48}$

$$
(\alpha h v)^{n}=A\left(h v-E_{\mathrm{g}}\right)
$$



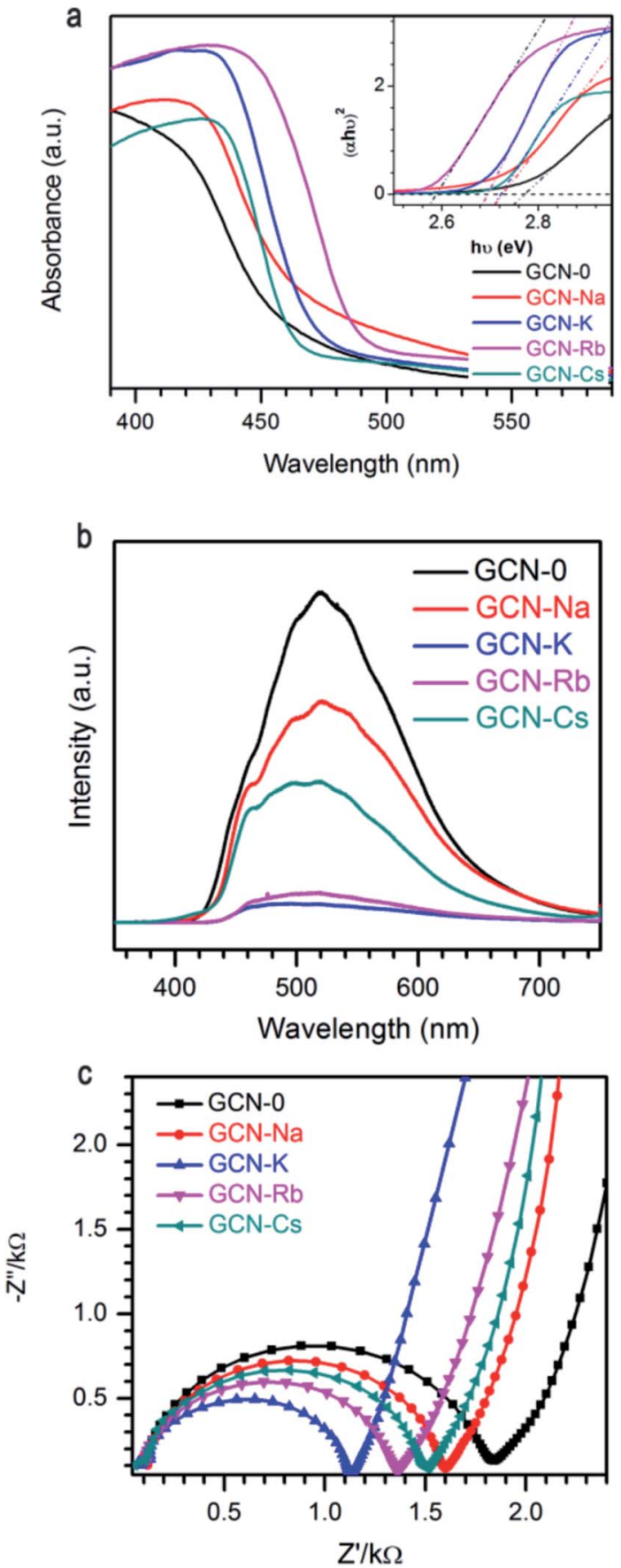

Fig. 5 (a) UV-visible spectra, (b) photoluminescence spectra, and (c) EIS Nyquist plot of $\mathrm{g}-\mathrm{C}_{3} \mathrm{~N}_{4}$ via $\mathrm{MCl}(\mathrm{M}=\mathrm{Na}, \mathrm{K}, \mathrm{Rb}, \mathrm{Cs})$ modification together with pristine $\mathrm{g}-\mathrm{C}_{3} \mathrm{~N}_{4}$.

where $A$ is a constant, $h$ is Plank's constant, $\nu$ is the frequency of incident light, $E_{\mathrm{g}}$ is the energy band gap, $\alpha$ is the absorption coefficient. $n=0.5$ for indirect band gap materials and $n=2$ for direct band gap material. Following the previous work of Liu et al., ${ }^{3}$ here we use $n=2$ and the inset of Fig. 3a shows a good linear fit. The $E_{\mathrm{g}}$ values determined by measuring the $x$-axis intercepts of the linear regime of $(\alpha h v)^{2}$ are 2.77, 2.73, 2.69, 2.59, and $2.72 \mathrm{eV}$ for GCN-0, GCN-Na, GCN-K, GCN-Rb, and GCN-Cs, respectively.
It is known that electrons will be excited from $\mathrm{VB}$ of $\mathrm{g}-\mathrm{C}_{3} \mathrm{~N}_{4}$ to $\mathrm{CB}$ under light irradiation, and then the photo-generate charge carriers can migrate to the surface to participate in photocatalytic reactions. Alternatively, the photo-induced electronhole pairs can recombine and emit light. Thus, photoluminescence (PL) spectroscopy is frequently used to probe the charge separation and recombination in photocatalysts. As shown in Fig. 5b, GCN-0 exhibits a strong and broad PL band centered at $\sim 515 \mathrm{~nm}$. It is found that PL intensity drops drastically in the sequence of GCN-K $>$ GCN-Rb $>$ GCN-Cs $>$ GCN $>$ $\mathrm{Na}$, where potassium chloride has the most pronounced effect on PL quenching. The much lower PL intensity of GCN-Na, GCN-K, GCN-Rb and GCN-Cs than GCN-0 indicates that the radiative recombination pathways associated with the longrange order of aromatic CN heterocycles were significantly
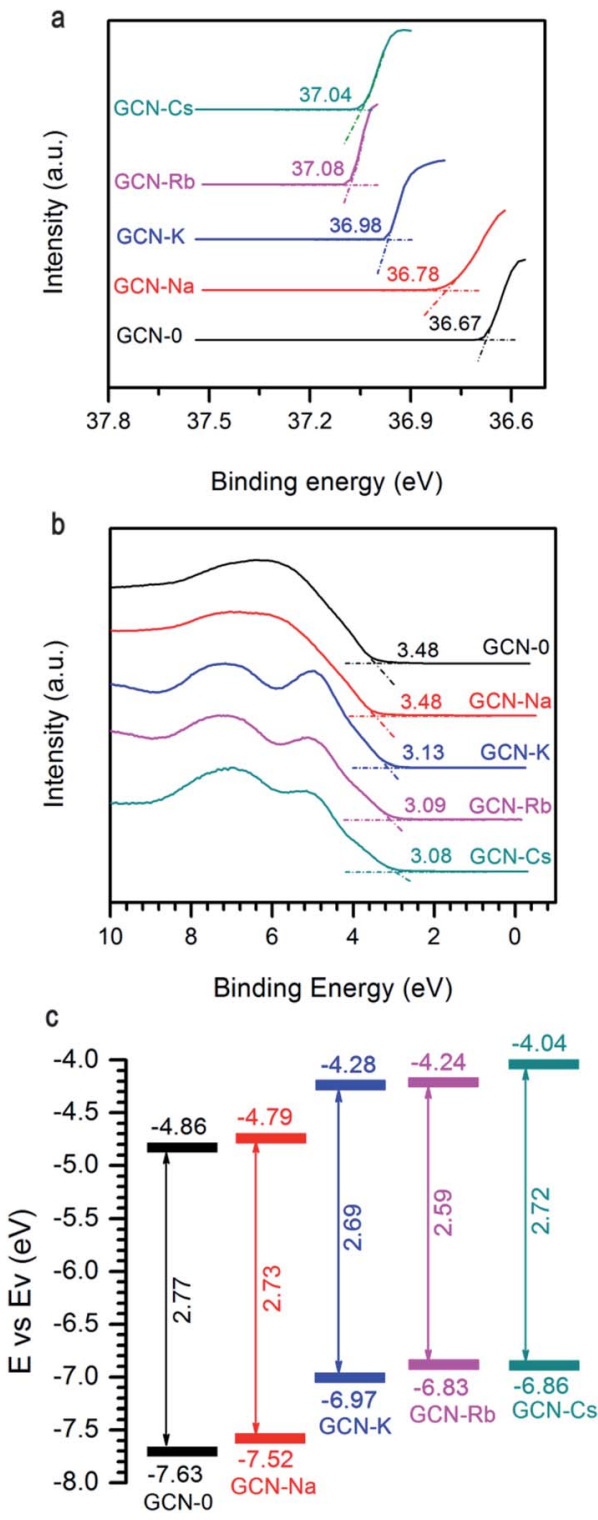

Fig. 6 (a) and (b) UPS of GCN-0, GCN-Na, GCN-K, GCN-Rb, and GCN-Cs. (c) Schematic of band gap and band offsets with respect to vacuum level. 
inhibited by alkali halides, especially $\mathrm{KCl}$. The effect of alkali halides on the charge carrier transfer and recombination behavior was further analyzed by electrochemical impedance spectra (EIS). Fig. 5c shows the Nyquist plots of GCN-0, GCN-Na, GCN-K, GCN-Rb and GCN-Cs. In general, the semicircle in the EIS spectra is ascribed to the contribution from charge transfer resistance $\left(R_{\mathrm{ct}}\right)$ and constant phase element (CPE) at the photocatalyst/ electrolyte interface, and the inclined line, resulting from the Warburg impedance, represents the ion-diffusion process in the electrolyte..$^{\mathbf{1 1 5 , 1 7 , 4 9 - 5 1}}$ From Fig. 5c, it can be seen that the radius of semicircle decreases in the order of GCN-K > GCN-Rb $>$ GCN-Cs $>$ GCN-Na $>$ GCN-0. This indicates that the recombination of electron and hole pairs in GCN-K is more effectively inhibited than that of GCN-0. Therefore, alkali halides result in red shifting of absorption edges and suppressed recombination of photogenerated electrons and holes in graphitic carbon nitride.

Another important factor affecting the performance of a photocatalyst is its $\mathrm{CB}$ and VB positions. Fig. 6a and b show the two ends of measured UPS spectra of GCN-0, GCN-Na, GCN$\mathrm{K}$, GCN-Rb and GCN-Cs. Generally, the ionization energy (equivalent to VB edge $\left(E_{\mathrm{v}}\right)$ ) of a semiconductor material can be estimated by subtracting the width of the HeII UPS spectra from the excitation energy $(40.8 \mathrm{eV})$ and the $\mathrm{CB}$ edge $\left(E_{\mathrm{c}}\right)$ can be estimated by $E_{\mathrm{v}}-E_{\mathrm{g}}$. Fig. $6 \mathrm{c}$ plots the extracted $E_{\mathrm{g}}$ and $\mathrm{CB} / \mathrm{VB}$ positions from DRS and UPS spectra. It is found that alkali halides can shift the CB and VB in upward directions, which is believed to be favorable for producing electrons with higher reducing-ability and strong-oxidation superoxide radicals under light irradiation.

The photocatalytic performance of all the samples was evaluated by degradation of $\mathrm{RhB}$ solutions under visible light irradiation, see Fig. 7a. When pristine $\mathrm{g}-\mathrm{C}_{3} \mathrm{~N}_{4}$ is used, $\sim 45 \%$ $\mathrm{RhB}$ could be decomposed subjected to $40 \mathrm{~min}$ light irradiation. Under the same conditions, the degradation efficiency of GCN$\mathrm{K}, \mathrm{GCN}-\mathrm{Rb}$ and GCN-Cs is $83 \%, 79 \%$ and $52 \%$, respectively. However, GCN-Na has lower photocatalytic degradation efficiency $\sim 16 \%$. As shown in Fig. $7 \mathrm{~b}$ and $\mathrm{c}$, the extracted reaction rate constants $k$ of $\mathrm{RhB}$ photodegradation are $15 \times 10^{-3}, 5 \times$ $10^{-3}, 44 \times 10^{-3}, 39 \times 10^{-3}$, and $17 \times 10^{-3} \mathrm{~min}^{-1}$ for GCN-0, GCN-Na, GCN-K, GCN-Rb, GCN-Cs, respectively. Therefore, alkali halides can improve the photocatalytic performance of graphitic carbon nitride except $\mathrm{NaCl}$. Among all the samples, $\mathrm{KCl}$ has the most pronounced effect. To understand the mechanism of this photocatalytic process, trapping experiment has been carried out on GCN-K. As shown in Fig. 7d, the degradation efficiency of RhB is significantly suppressed when benzoquinone (BQ, $2 \mathrm{mM}$, as a superoxide radicals ${ }^{\circ} \mathrm{O}_{2}{ }^{-}$scavenger) was added. This means that the ${ }^{\circ} \mathrm{O}_{2}{ }^{-}$acts as the main active specie in the photocatalytic degradation. It is plausible that alkali halides modify the microstructure of the obtained graphitic carbon nitride, which in turn affects the charge carrier transfer behavior and reduces their radiative recombination. Moreover, the structure modification also results in enhanced visible-light absorption capability and improved reduction
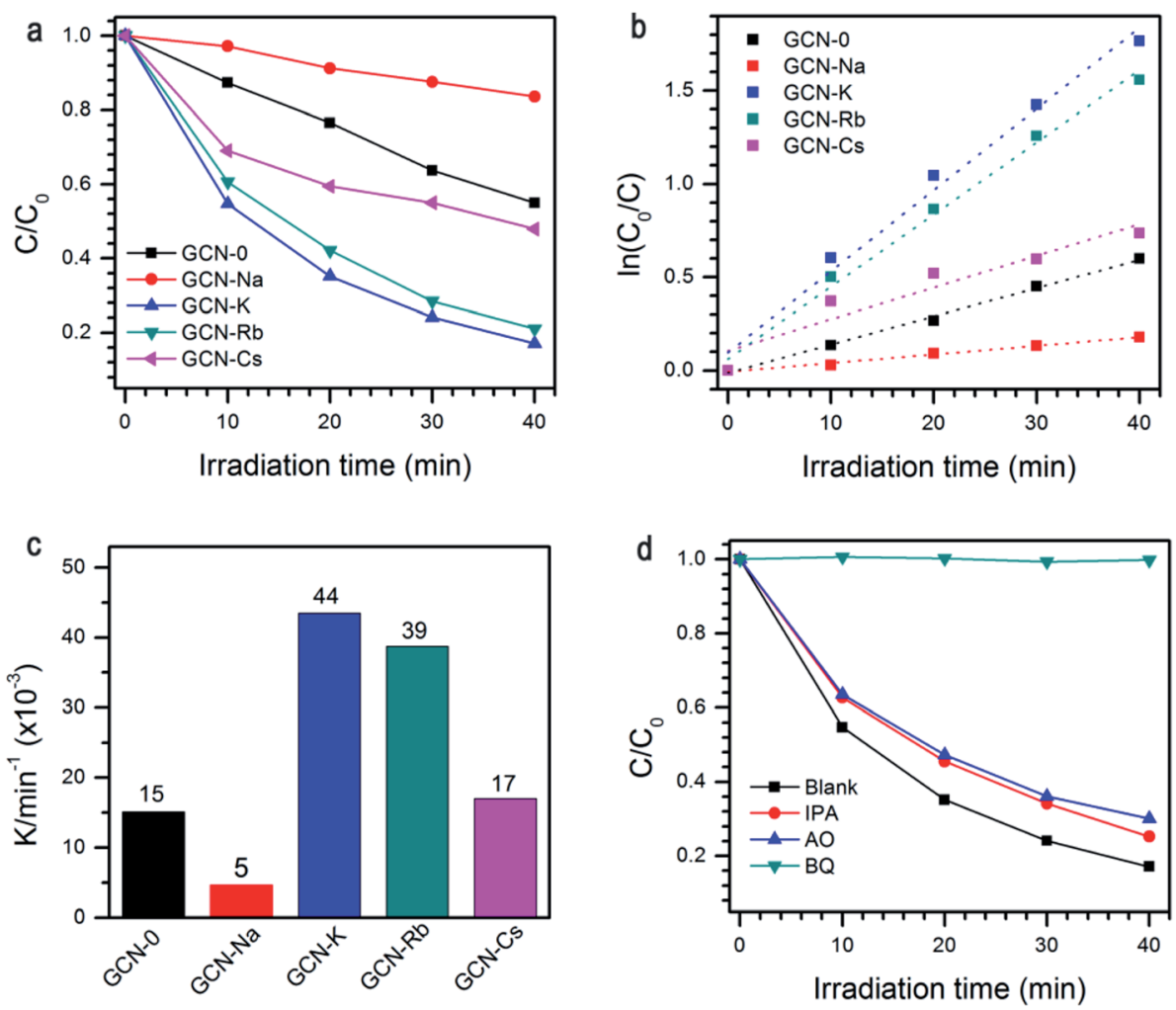

Fig. 7 (a) Photocatalytic degradation of RhB by GCN-O, GCN-Na, GCN-K, GCN-Rb and GCN-Cs under visible-light irradiation; (b) first order kinetic fitting data and (c) rate constant $k$ for the photodegradation of RhB by GCN-0, GCN-Na, GCN-K, GCN-Rb and GCN-Cs; (d) GCN-K with adding scavengers of $B Q, I P A$, and $A O$. 


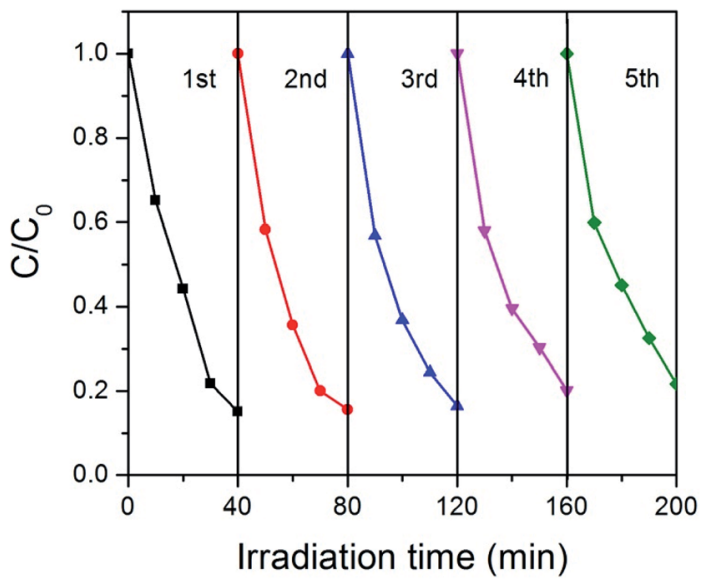

Fig. 8 Photocatalytic degradation of RhB on GCN-K for five cycles.

ability of electrons. Therefore, alkali modified samples can have higher photodegradation efficiency than pristine $\mathrm{g}-\mathrm{C}_{3} \mathrm{~N}_{4}$. Since the structure modification depends on the type of cationic ions, the changes of photocatalytic activity is also related to cationic ions. We note that $\mathrm{NaCl}$ has the least effect on the structural, electronic, and optical modification of $\mathrm{g}-\mathrm{C}_{3} \mathrm{~N}_{4}$ but much smaller $S_{\text {BET }}$ (10.44vs. $1.92 \mathrm{~m}^{2} \mathrm{~g}^{-1}$ ), which might be responsible for the observed decreasing of photocatalytic activity.

Stability is very important for the practical applications of photocatalysts. For this purpose, we tested the cycle stability of GCN-K and plotted the results in Fig. 8. Apparently, there is no clear deterioration of the photocatalytic capability after 5 recycles, indicating that GCN-K has good stability.

\section{Conclusions}

In summary, we investigated the effect of alkali halides on the structural, electronic, optical, and photocatalytic properties of graphitic carbon nitride and compare the effects of different cationic ions. It is found that $\mathrm{g}-\mathrm{C}_{3} \mathrm{~N}_{4}$ photocatalysts synthesized from the mixture of melamine and alkali halides have reduced in-plane long-range periodic ordering, and larger cationic ions have more pronounced effect. This alkali halide salt-induced structural modification facilitates separation and transfer of photogenerated electrons and holes, reduces band gap energies and shifts CB and VB edges in upward direction. The cationdependent changes of structural, electronic and optical properties result in different photocatalytic performance of GCN-0, GCN-Na, GCN-K, GCN-Rb, and GCN-Cs.

\section{Acknowledgements}

This work was supported by the National Natural Science Foundation of China [Grant No. 51402277, 51402274, and 21401180].

\section{References}

1 W.-J. Ong, L.-L. Tan, Y. H. Ng, S.-T. Yong and S.-P. Chai, Chem. Rev., 2016, 116, 7159-7329.
2 M. Kapilashrami, Y. Zhang, Y. S. Liu, A. Hagfeldt and J. Guo, Chem. Rev., 2014, 114, 9662-9707.

3 J. Liu, Y. Liu, N. Liu, Y. Han, X. Zhang, H. Huang, Y. Lifshitz, S.-T. Lee, J. Zhong and Z. Kang, Science, 2015, 347, 970-974.

4 Y. Ma, X. Wang, Y. Jia, X. Chen, H. Han and C. Li, Chem. Rev., 2014, 114, 9987-10043.

5 D. J. Martin, K. P. Qiu, S. A. Shevlin, A. D. Handoko, X. Chen, Z. X. Guo and J. W. Tang, Angew. Chem., Int. Ed., 2014, 53, 9240-9245.

6 A. Fujishima and K. Honda, Nature, 1972, 238, 37-38.

7 A. Iwase, S. Yoshino, T. Takayama, Y. H. Ng, R. Amal and A. Kudo, J. Am. Chem. Soc., 2016, 138, 10260-10264.

8 D. Ma, J. Wu, M. Gao, Y. Xin and C. Chai, Chem. Eng. J., 2017, 316, 461-470.

9 Y. Yuan, H. Lu, Z. Ji, J. Zhong, M. Ding, D. Chen, Y. Li, W. Tu, D. Cao, Z. Yu and Z. Zou, Chem. Eng. J., 2015, 275, 8-16.

10 A. Thomas, A. Fischer, F. Goettmann, M. Antonietti, J.-O. Müller, R. Schlögl and J. M. Carlsson, J. Mater. Chem., 2008, 18, 4893-4908.

11 Z.-F. Huang, J. J. Song, L. Pan, Z. M. Wang, X. Q. Zhang, J.-J. Zou, W. B. Mi, X. W. Zhang and L. Wang, Nano Energy, 2015, 12, 646-656.

12 Z. Z. Lin and X. C. Wang, Angew. Chem., Int. Ed., 2013, 52, 1735-1738.

13 J. Gao, J. Wang, X. Qian, Y. Dong, H. Xu, R. Song, C. Yan, H. Zhu, Q. Zhong, G. Qian and J. Yao, J. Solid State Chem., 2013, 228, 60-64.

14 P. Niu, L. L. Zhang, G. Liu and H.-M. Chen, Adv. Funct. Mater., 2012, 22, 4763-4770.

15 P. Xia, B. Zhu, J. Yu, S. Cao and M. Jaroniec, J. Mater. Chem. A, 2017, 5, 3230-3238.

16 Y. Yang, J. Chen, Z. Mao, N. An, D. Wang and B. D. Fahlman, RSC Adv., 2017, 7, 2333-2341.

17 L.-H. Lin, H. H. Ou, Y. F. Zhang and X. C. Wang, ACS Catal., 2016, 6, 3921-3931.

18 X. Liu, K. Xu, P. Chen, K. Jia, S. Liu and C. Wu, J. Mater. Chem. A, 2014, 2, 18924-18928.

19 J. Yan, C. Zhou, P. Li, B. Chen, S. Zhang, X. Dong, F. Xi and J. Liu, Colloids Surf., A, 2016, 508, 257-264.

20 K. Li, S. Gao, Q. Y. Wang, H. Xu, Z. Y. Wang, B. B. Huang, Y. Dai and J. Lu, ACS Appl. Mater. Interfaces, 2015, 7, 90239030.

21 Z. P. Yang, Z. J. Sun, X. Liu, H. X. Jia and P. W. Du, Nanoscale, 2016, 8, 4748-4756.

22 Q. Zhang, H. Wang, S. Chen, Y. Su and X. Quan, RSC Adv., 2017, 7, 13223-13227.

23 Z. Zhang, M. Wang, W. Cui and H. Sui, RSC Adv., 2017, 7, 8167-8177.

24 J. Wang, H. Xu, X. Qian, Y. Dong, J. Gao, G. Qian and J. Yao, Chem.-Asian J., 2015, 10, 1276-1280.

25 L. Yang, J. Huang, L. SHi, L. Cao, Q. Yu, Y. Jie, J. Fei, H. Ouyang and J. Ye, Appl. Catal., B, 2017, 204, 335-345.

26 P. Niu, L. Zhang, G. Liu and H.-M. Cheng, Adv. Funct. Mater., 2012, 22, 4763-4770.

27 Y. Kang, Y. Yang, L.-C. Yin, X. Kang, L. Wang, G. Liu and H.-I. Cheng, Adv. Mater., 2016, 28, 6471-6477. 
28 Y. Kang, Y. Yang, L.-C. Yin, X. Kang, G. Liu and H.-M. Cheng, Adv. Mater., 2015, 27, 4572-4577.

29 L. Lin, H. Ou, Y. Zhang and X. Wang, ACS Catal., 2016, 6, 3921-3931.

30 W. Fang, J. Liu, L. Yu, Z. Jiang and W. Shangguan, Appl. Catal., B, 2017, 209, 631-636.

31 S. Hu, F. Li, Z. Fan, F. Wang, Y. Zhao and Z. Lv, Dalton Trans., 2015, 44, 1084-1092.

32 M. Zhang, X. Bai, D. Liu, J. Wang and Y. Zhu, Appl. Catal., B, 2015, 164, 77-81.

33 Y. Li, H. Xu, S. Ouyang, D. Lu, X. Wang, D. Wang and J. Ye, J. Mater. Chem. A, 2016, 4, 2943-2950.

34 H. H. Liu, D. C. Chen, Z. Q. Wang, H. J. Jing and R. Zhang, Appl. Catal., B, 2017, 203, 300-313.

35 Y. X. Li, S. Ouyang, H. Xu, X. Wang, Y. P. Bi, Y. F. Zhang and J. H. Ye, J. Am. Chem. Soc., 2016, 138, 13289-13297.

36 H. Yu, S. Run, Y. Zhao, T. Bian, Y. Zhao, C. Zhou, G. I. N. Waterhouse, L.-Z. Wu, C.-H. Tung and T. Zhang, Adv. Mater., 2017, 29, 1605148.

37 J. Zhang, S. Hu and Y. Wang, RSC Adv., 2014, 4, 62912-62919.

38 C. Y. Liu, Y. H. Zhang, F. Dong, A. H. Reshak, L. Q. Ye, N. Pinna, C. Zeng, T. R. Zhang and H. W. Huang, Appl. Catal., B, 2017, 203, 465-474.

39 Q. Xiang, J. Yu and M. Jaroniec, J. Phys. Chem. C, 2011, 115, 7355-7363.

40 Y. Shang, X. Chen, W. Liu, P. Tan, H. Chen, L. Wu and C. Ma, Appl. Catal., B, 2017, 204, 78-88.
41 X. L. Wang, W. Q. Fang, H. F. Wang, H. Zhang, H. Zhao, Y. Yao and H. G. Yang, J. Mater. Chem. A, 2013, 1, 1408914096.

42 P. V. Zinin, L.-C. Ming, S. K. Sharma, V. N. Khabashesku, X. Liu, S. Hong, C. Endo and T. Acosta, Chem. Phys. Lett., 2009, 472, 69-73.

43 H. Lan, L. Li, X. An, F. Liu, C. Chen, H. Liu and J. Qu, Appl. Catal., B, 2017, 204, 49-57.

44 J. Li, B. Shen, Z. Hong, B. Lin, B. Gao and Y. Chen, Chem. Commun., 2012, 48, 12017-12019.

45 H. Ma, Y. Li, S. Li and N. Liu, Appl. Surf. Sci., 2015, 357, 131138.

$46 \mathrm{Y} . \mathrm{Bu}$ and Z. Chen, Electrochim. Acta, 2014, 144, 42-49.

47 H. H. Park, B. H. Kim, S. H. Song, J. Kwon, B. S. Kong, K. Kang and S. Jeon, Nano Lett., 2012, 12, 2871-2876.

48 J. Navas, A. Sanchez-Coronilla, T. Aguilar, N. C. Hernandez, D. M. de los Santos, J. Z. Sanchez-Marquez, D. C. Fernandez-Lorenzo, R. Alcantaraa and J. MartinCalleja, Phys. Chem. Chem. Phys., 2014, 16, 3835-3845.

49 Z. Zhao, Y. Sun, Y. Zhang and H. ZHao, RSC Adv., 2015, 5, 39549-39556.

50 X. J. She, L. Liu, H. Y. Ji, Z. Mo, Y. P. Li, L. Y. Huang, D. L. Du and H. Xu, Appl. Catal., B, 2016, 187, 144-153.

51 J. Xu, L. Zhang, R. Shi and Y. ZHu, J. Mater. Chem. A, 2013, 1, 14766-14772. 